\title{
Proof of concept for the clinical utility of a convection enhanced delivery platform for immuno-oncology drugs against brain tumors
}

\author{
Fatih M Uckun*, Sanjive Qazi and Vuong N Trieu \\ Immuno-Oncology Program, Oncotelic Inc, Agoura Hills, CA; Ares Pharmaceuticals, St. Paul, MN, USA
}

\begin{abstract}
Here we present a post-hoc analysis of the long-term follow-up data for the Phase IIB study NCT00431561 regarding the clinical activity of the TGFbeta2-specific RNA therapeutic OT101/Trabedersen in recurrent/refractory WHO Grade 3 anaplastic astrocytoma (R/R AA) patients. OT101 was administered intratumorally by continuous infusion via CEDOT for 5 consecutive months to 27 recurrent/refractory WHO Grade 3 anaplastic astrocytoma (R/R AA) patients in two dose cohorts $(2.5 \mathrm{mg} /$ cycle and $19.8 \mathrm{mg} / \mathrm{cycle}) .14$ of the 27 patients $(51.9 \%)$ had a sustained partial response (PR) or complete response (CR). The median overall survival (OS) time of 1136 days was significantly better than the 590 days median OS ( $\log \operatorname{Rank} \boldsymbol{\chi}^{2}=6.5, \mathrm{P}$-value $\left.=0.011\right)$ of the temozolomide (TMZ)-treated control patient population $(\mathrm{N}=11)$.
\end{abstract}

\section{Introduction}

Prognosis of high-grade gliomas (HGG) has not significantly improved despite recent advances in neurosurgery, chemotherapy, immuno-oncology, and radiation therapy [1-4] Therefore, effective salvage therapies are needed for recurrent/refractory HGG patients who have failed their standard therapy options [5-7]. Immuno-oncology drugs, including the immune-check point inhibitors (ICI) often have a delayed onset of action and have limited applicability for brain tumor therapy due to their highly variable central nervous system penetration caused by the blood brain barrier (BBB) [8-10]. Convection-enhanced delivery (CED) is a strategy that bypasses the BBB entirely and enhances drug distribution by applying hydraulic pressure to deliver agents directly and evenly into a target region. This technique reliably distributes the drug homogenously through the interstitial space of the target region and achieves high local drug concentrations in the brain [11]. The CEDOT platform was designed to maximize the clinical benefit of immuno-oncology drugs for aggressive brain tumors. The CEDOT platform that allows repeated infusions of immuno-oncology drugs in an outpatient setting for desired extended periods of time, includes a single stereotactically placed silicone-based biocompatible intratumoral microcatheter, a subcutaneously implanted port access system, and a portable external pump.

The experimental immuno-oncology drug OT101 (also known as Trabedersen), a TGF $\beta 2$-specific synthetic antisense oligodeoxynucleotide (S-ODN), is a first-in-class RNA therapeutic with an FDA orphan drug designation and pediatric rare disease designation, that is designed to abrogate the tumor-promoting and immunosuppressive actions of TGF $\beta 2$ in aggressive brain tumors [1214]. OT101 has been shown to (i) reduce TGF $\beta 2$ production/secretion, (ii) inhibit proliferation as well as invasive migration, and (iii) enhance sensitivity to lymphocyte-mediated cytotoxicity of human HGG cells
[12-14]. Intracerebral infusion of OT101 results in rapid distribution of OT101 from the infusion site to other parts of the brain, including remaining cerebrum, cerebellum, pineal body, and spinal cord in preclinical studies [15]. The feasibility of intratumoral application of OT101 for treatment of recurrent/refractory HGG patients was first shown in Phase I clinical trials that also provided early activity signals $[13,14]$. The preliminary findings of a Phase II study (NCT00431561) confirmed its favorable safety profile and showed that OT101 can offer early disease control to R/R high-grade glioma patients at 6 months at a rate comparable to that achieved with the standard alkylating chemotherapy drug temozolomide [16]. We are now reporting our post-hoc analysis of the long-term follow-up data on the R/R AA patient subpopulation treated in the NCT00431561 study as proof of concept for the clinical utility of the CEDOT platform. Notably, OT101 administered intratumorally via the CEDOT platform exhibited clinically meaningful single-agent activity and induced durable complete response (CR), partial response (PR) in more than half of the treated $\mathrm{R} / \mathrm{R}$ AA patients. The median overall survival of patients receiving the CEDOT-delivered experimental therapy 1136 (95\% CI: 811 - 1743) days which was significantly better than the 590 (95\% CI: 287 - NA) days median OS (Log Rank $\chi^{2}=6.5$, P-value=0.011) of the TMZ-treated patient population.

${ }^{\star}$ Correspondence to: Fatih M. Uckun, Immuno-Oncology Program, Oncotelic Inc, Agoura Hills, CA; Ares Pharmaceuticals, St. Paul, MN, USA, E-mail: fatihuckun53@gmail.com

Key words: RNA therapeutic, glioma, convection-enhanced delivery, anaplastic astrocytoma, immuno-oncology

Received: December 04, 2019; Accepted: December 27, 2019; Published: December 31, 2019 


\section{Materials and methods}

\section{Study approval}

This study was performed in compliance with the $\mathrm{ICH}(\mathrm{E} 6)$ good clinical practice (GCP) guidelines and with approval from independent ethics committees as well as Institutional Review Boards of the participating institutions. Each patient provided a written informed consent. An independent Data and Safety Monitoring Board participated in the review of review of the clinical data. The trial was registered with ClinicalTrials.gov (Study No. NCT00431561). The study was subject to all applicable regional and national regulations. Monitoring of the German, Austrian and Israeli centres was performed by the Clinical Research Organizations (CROs) HESPERION Ltd., Switzerland and Premier Research Group plc, United Kingdom (UK). The Indian centres were monitored by the CRO SIRO Clinpharm Private Ltd. The CRO Evidence CPR monitored the study sites in Russia and Georgia. Data management was performed by Premier Research Group plc. in Crowthorne, UK, with data entry being performed in Darmstadt, Germany.

\section{Investigational medicinal product and CED drug delivery system}

Randomized groups of two OT101 dose cohorts received OT101 via a single intratumoral catheter that was implanted into target lesion. OT101 was infused intratumorally using CED. One treatment cycle with OT101 lasted 14 days and consisted of a 7-day administration of
OT101, followed by administration of isotonic saline solution for 7 days. The port system and the intratumoral catheter for OT101 delivery were implanted 2 days prior to starting the OT101 treatments and removed after the last scheduled 7-day infusion of isotonic (0.9\%) saline. OT101 dissolved in isotonic $(0.9 \%)$ aqueous sodium chloride solution at a final concentration of either $10 \mu \mathrm{M}$ or $80 \mu \mathrm{M}$ was administered at 4 $\mu \mathrm{L} / \mathrm{min}$ for 7 days. The total OT101 dose per cycle was $2.5 \mathrm{mg}(10 \mu \mathrm{M}$ group) or $19.8 \mathrm{mg}$ ( $80 \mu \mathrm{M}$ group). According to the clinical protocol, eligible patients assigned to an OT101 dose cohort were to be treated with OT101 for at least 8 weeks corresponding to 4 cycles of OT101 and receive a maximum of 11 treatment cycles of OT101. The investigational medicinal product (IMP) was provided to treating centers as a sterile lyophilizate in $50 \mathrm{~mL}$ glass vials containing $7.37 \mathrm{mg}$ OT101. Prior to administration the lyophilized OT101 was reconstituted in sterile $0.9 \%$ isotonic sodium chloride solution for infusion. A concentration of either $10 \mu \mathrm{M}$ or $80 \mu \mathrm{M}$ of active ingredient dissolved in isotonic (0.9\%) aqueous sodium chloride solution was administered. The OT101 dose delivered each cycle was either $2.5 \mathrm{mg}$ or $19.8 \mathrm{mg}$. During the treatmentfree intervals, blocking of the intratumoral catheter was prevented by a continuous isotonic saline infusion. Saline infusion started at a flow rate of $4 \mu \mathrm{L} / \mathrm{min}$ to rinse the remaining OT101 solution in the catheter. After $10 \mathrm{~h}$, the flow rate was reduced to $1 \mu \mathrm{L} / \mathrm{min}$ for the duration of the 7-day infusion period.

The CED system "CEDOT" depicted in Figure 1 included the following components: An implanted catheter placed intratumorally
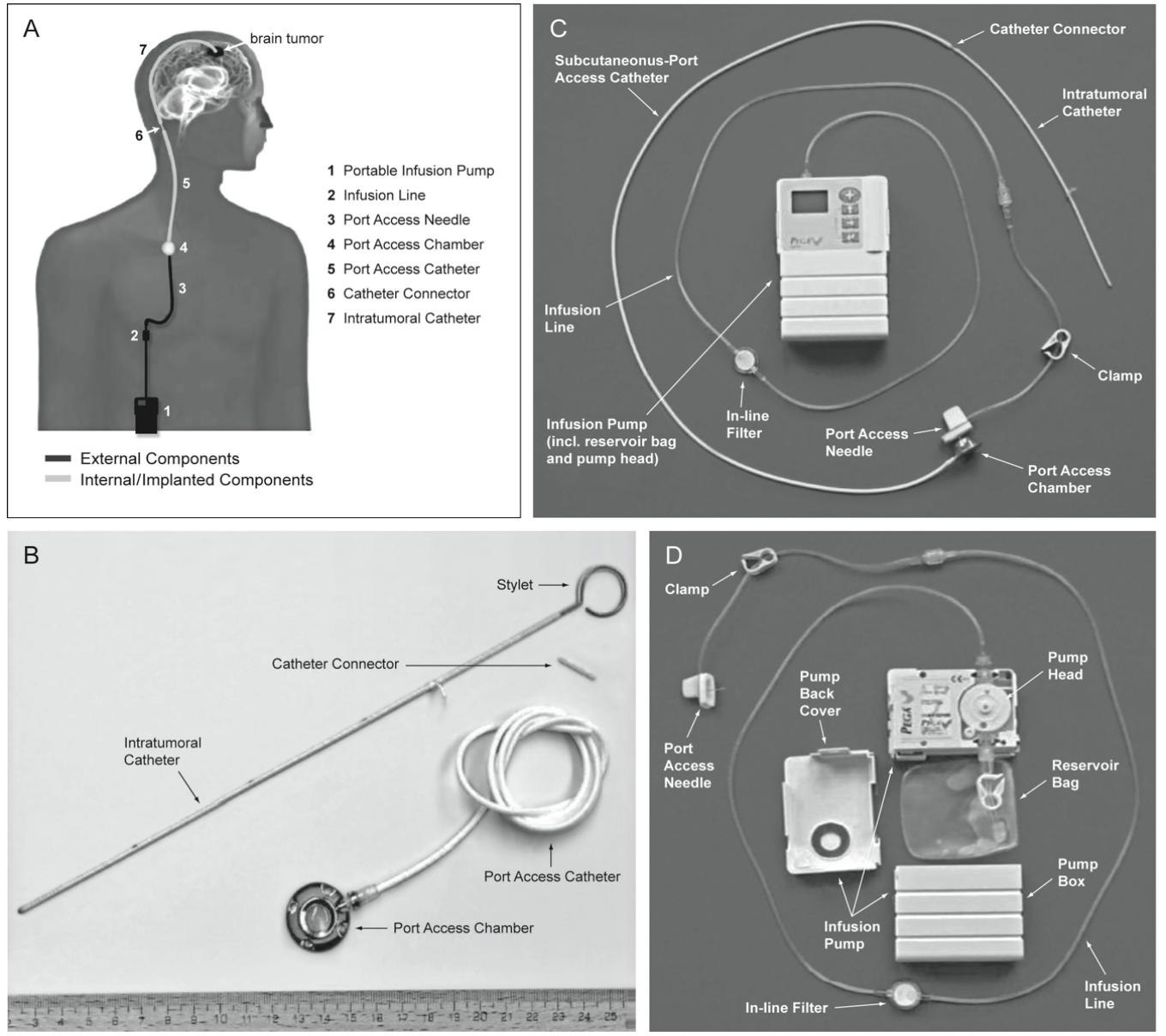

Figure 1. Components of the CEDOT system for intratumoral OT101 therapy. [A] Overview. [B] Implanted components. [C] CED system assembled. [D] external components 
and connected subcutaneously to a port access system (consisting of port chamber and port catheter) by a connecting piece. For this purpose, a connecting piece was used between port catheter and ventricular catheter. Both catheters were barium impregnated to facilitate control of their correct placement. The port system was pre-filled with isotonic $(0.9 \%)$ saline solution and its postoperative position as well as the localization of the intratumoral catheter tip was controlled and documented by X-ray and CT. A portable external pump (Pegasus Vario, with study-specific configuration) (Venner Medical, Dänischenhagen, Germany) ensured the delivery of the drug at the specified infusion rate of $4 \mu \mathrm{L} / \mathrm{min}$. The external portable pump was connected to the port system by a special port puncture needle. The subcutaneous access system was implanted by local surgeons or neurosurgeons. The intratumoral catheter was placed by the neurosurgeons. Cranial CT (CCT) or brain MRI were performed during the preparation period for determination of the target region within the brain tumor for placement of the intratumoral catheter and calculation of the planned position of the catheter tip. A CCT scan was performed on Day 2 after placement of the intratumoral catheter to ensure correct placement of the catheter tip as well as to detect any possible procedure-related complication (e.g. hemorrhage). The local neuroradiologists, radiologists, and their technical assistants obtained MRIs and CCTs. The described delivery system allowed treatment of eligible patients repeated OT101 infusions in an outpatient setting.

\section{Efficacy measurements}

Activity and efficacy analyses were performed for all 27 patients in the OT101 treatment groups. For immediate decision-making during the course of the study, the local neuroradiologists evaluated patients local MRIs according to study specific procedures, filed in the TMF. For a standardized response assessment for the study analysis, an independent Central MRI Reading (CMRIR) was performed by a specialized central reading institute (Timaq Medical Imaging Inc, Zurich, Switzerland). Central reading was conducted by two independent neuroradiologists with an additional adjudicator for cases of predefined discrepancies in the reports of the two readers.

The axial T2- and T1-weighted sequences were performed in identical slice positions to ensure comparability. The coronal scans were oriented parallel to the dorsal contour of the brain stem at the level of the pons. The sagittal T1-weighted 3D sequence covered the whole brain. Before the IV injection of contrast medium (CM), the acquisition sequence included T2 axial, native (Turbo spin echo/TSE or fast spin echo/FSE, slice thickness: $6 \mathrm{~mm}$, Gap: $0.6 \mathrm{~mm}$, TE/Echo time: $80-120 \mathrm{msec}$ ) and T1 axial native (spin echo/SE, not TSE, slice thickness: $6 \mathrm{~mm}$, Gap: $0.6 \mathrm{~mm}$, TE/ Echo time: $80-120 \mathrm{msec}$ ) images. One minute after intravenous injection of Gadolinium-based CM (0.1 $\mathrm{mmol} / \mathrm{kg}$ body weight), the acquisition sequence included $\mathrm{T} 1$ axial + CM (SE, not TSE; slice thickness: $6 \mathrm{~mm}$; gap: $0.6 \mathrm{~mm}$, TE: $12-20 \mathrm{msec}$ ), T1 coronal + CM (SE, not TSE; slice thickness: $6 \mathrm{~mm}$; gap: 1.2-1.8 mm, TE: $12-20 \mathrm{msec}$ ) and T1-3D gradient echo, sagittal + CM (to cover the whole brain, slice thickness: maximum $1.5 \mathrm{~mm}$ ) images.

Best overall response (BOR) was defined as the best response (i.e. CR, PR or SD) observed from the start of treatment until disease progression. For determining the treatment response of individual patients to OT101, standard MacDonald criteria were used. Complete Response (CR) was defined as the disappearance of all enhancing tumor on consecutive MRIs (at least one month apart), off steroids. Partial Response (PR) was defined as $\geq 50 \%$ reduction in size of enhancing tumor on consecutive MRIs (at least one month apart), steroids stable or reduced. CR and PR were confirmed by two consecutive observations not less than four weeks apart.

Duration of objective response was defined as the interval from the onset of CR or PR to SD, PD or death due to any cause, whichever occurred first. Patients who did not progress or die were censored at the last tumor assessment date. Time to progression (TTP) was calculated for all patients from the date of randomization to the date of the first documented tumor progression. Patients who were switched to another anti-tumor therapy were assumed progressed at the time of switch even if the progression was not documented by MRI assessments. Patients who remained alive without PD were censored at last follow-up. If the patient had not shown clinical signs of progression, continuation of study treatment was allowed in case of tumor progression according to MRI assessment within the first three months.

Overall survival (OS) was the time from the date of randomization to time of death. Surviving patients were censored at their last followup. Progression-free survival (PFS) was the time from randomization to documentation of PD or death. Patients who remained alive without PD were censored at last follow-up. Standard definitions were used for time to progression and duration of objective response.

\section{Patient characteristics and execution of the clinical trial}

NCT00431561 was a multi-national, multi-center, open-label interventional clinical study. The diagnosis was histopathologically confirmed before start of treatments. Patients had to have an expected life expectancy of $\geq 3$ months and a baseline KPS score $\geq 70 \%$. Patients who had recent tumor resection within 14 days prior to study entry were excluded as were patients receiving radiation therapy within eight weeks prior to randomization. Treatment with chemotherapy, hormone therapy, or any other therapies with established or suggested antitumor effects had to be finished 4 weeks to 6 weeks (nitrosureas only) before randomization. No prior stereotactic radiosurgery or interstitial brachytherapy and no TGF $\beta 2$-targeting therapy or antitumor vaccination were allowed. Patients who had received another investigational agent within 30 days prior to randomization were not eligible. In order to isolate the clinical single agent anti-HGG activity of OT101, no other cancer treatments, standard or experimental (including but not limited to radiation therapy, chemotherapy, or immunotherapy) were administered unless the patient experienced progression of disease (PD). In the NCT00431561 study, a total of 98 patients (AA: 30; GBM: 68) were randomized to one of the 2 treatment arms of OT101 representing 2 different dose cohorts, namely $2.5 \mathrm{mg} /$ cycle $(\mathrm{N}=48)$ and $19.8 \mathrm{mg} /$ cycle $(\mathrm{N}=50)$, respectively. Of the $30 \mathrm{AA}$ patients, 14 were randomized to the low dose cohort and 16 were randomized to the high dose cohort. A total of 3 patients ( 2 in the low dose cohort and 1 in the high dose cohort) discontinued the study after randomization but before any intervention. The remaining 27 patients, 12 in the low dose cohort and 15 in the high dose cohort served both as the safety population as well as the modified intent to treat population (Table 1). These 27 patients as well as 11 control patients who represent all AA patients who were treated with the standard chemotherapy drug TMZ represent the study population for this post-hoc analysis. Patient characteristics and the neuro-oncologic medical history of the patients are shown in Table 2. There were no statistically significant differences between the OT101-treated $(\mathrm{N}=27)$ and TMZ-treated $(\mathrm{N}=11)$ patient populations (Table 2) except for their objective response rates. In particular, their age, gender/race distribution, performance status, target lesion size, previous treatment history, and time from previous therapy were similar. 
Table 1. Analysis populations of AA patients randomized to treatment with OT101

\begin{tabular}{|c|c|c|c|}
\hline Treatment group & $\begin{array}{c}\text { OT101 } \\
2.5 \mathrm{mg} / \text { cycle } \\
\mathrm{N}\end{array}$ & $\begin{array}{c}\text { OT101 } \\
\text { 19.8mg/cycle } \\
\text { N }\end{array}$ & $\begin{array}{c}\text { Combined Total } \\
\mathbf{N}\end{array}$ \\
\hline \multicolumn{4}{|l|}{ Patient population } \\
\hline Intent-to-Treat (ITT) & 14 & 16 & 30 \\
\hline Discontinued after randomization (before catheter-port surgery in OT101 groups) & 2 & 1 & 3 \\
\hline Safety population (Catheter surgery and/or OT101 treatment) & 12 & 15 & 27 \\
\hline Discontinued after catheter surgery, before OT101 administration & 0 & 0 & 0 \\
\hline Modified Intent to Treat population (mITT)/Treated with OT101 & 12 & 15 & 27 \\
\hline
\end{tabular}

N: Number of patients; AA: Anaplastic astrocytoma (WHO Grade 3)

Table 2. Patient information and objective response rates OT101 (N=27) TMZ $(\mathrm{N}=11)^{*}$

\begin{tabular}{|c|c|c|c|}
\hline $\begin{array}{l}\text { Gender - \# (\%) } \\
\text { Female } \\
\text { Male }\end{array}$ & $\begin{array}{c}8(29.6) \\
19(70.4)\end{array}$ & $\begin{array}{l}4(36.4) \\
7(63.6)\end{array}$ & NS \\
\hline $\begin{array}{l}\text { Race - \# (\%) } \\
\text { Caucasian } \\
\text { Asian } \\
\text { Black }\end{array}$ & $\begin{array}{c}13(48.1) \\
14(51.9) \\
0(0.0)\end{array}$ & $\begin{array}{l}3(27.3) \\
8(72.7) \\
0(0.0)\end{array}$ & NS \\
\hline $\begin{array}{l}\text { Age (Years) } \\
\text { Median (Range) } \\
\text { Mean } \pm \text { SE }\end{array}$ & $\begin{array}{l}40(23-59) \\
40.3 \pm 2.0\end{array}$ & $\begin{array}{l}39(21-67) \\
39.7 \pm 4.2\end{array}$ & NS \\
\hline $\begin{array}{l}\text { KPS Score at Baseline } \\
\text { Median (Range) } \\
\text { Mean } \pm \text { SE }\end{array}$ & $\begin{array}{c}90(70-100) \\
87.0 \pm 1.8\end{array}$ & $\begin{array}{c}90(70-100) \\
87.3 \pm 2.7\end{array}$ & NS \\
\hline $\begin{array}{l}\text { Size of Largest Target Tumor Lesion } \\
\text { 2-D in } \mathrm{cm}^{2}-\text { Median }(\text { Mean } \pm \text { SE) } \\
\text { 3-D in } \mathrm{cm}^{3}-\text { Median }(\text { Mean } \pm \text { SE) }\end{array}$ & $\begin{array}{c}7.6(7.6 \pm 0.7) \\
17.3(19.0 \pm 2.0)\end{array}$ & $\begin{array}{c}8.5(8.6 \pm 1.3) \\
13.4(23.2 \pm 5.5)\end{array}$ & NS \\
\hline $\begin{array}{l}\text { OT101 Dose Cohort - \# (\%) } \\
\text { Low }(2.5 \mathrm{mg} / \text { cycle }) \\
\text { High }(19.8 \mathrm{mg} / \text { cycle })\end{array}$ & $\begin{array}{l}12(44.4) \\
15(55.6)\end{array}$ & NA & NA \\
\hline $\begin{array}{l}\text { Number of OT101 Cycles } \\
\text { Median (Range) } \\
\text { Mean } \pm \text { SE }\end{array}$ & $\begin{array}{c}11(2-11) \\
9.0 \pm 0.5\end{array}$ & NA & NA \\
\hline $\begin{array}{l}\text { Total OT101 Dose }\left(\mathbf{m g} / \mathbf{m}^{2}\right) \\
\text { Median (Range) } \\
\text { Mean } \pm \text { SE }\end{array}$ & $\begin{array}{l}39.6(5.9-152.1) \\
\quad 61.2 \pm 9.9\end{array}$ & NA & NA \\
\hline $\begin{array}{l}\text { Number of Chemotherapy Cycles } \\
\text { Median (Range) } \\
\text { Mean } \pm \text { SE }\end{array}$ & $\begin{array}{l}\text { NA } \\
\text { NA }\end{array}$ & $\begin{array}{l}6(1-6) \\
5.1 \pm 0.5\end{array}$ & NA \\
\hline $\begin{array}{l}\text { Time from last cancer therapy } \\
\text { Median }(M e a n \pm S E)\end{array}$ & $154(449 \pm 153)$ & $105(211 \pm 81)$ & NS \\
\hline $\begin{array}{l}\text { Time from Diagnosis } \\
\text { Median }(\text { Mean } \pm \text { SE) }\end{array}$ & $251(608 \pm 183)$ & $224(459 \pm 150)$ & NS \\
\hline \multicolumn{4}{|l|}{ Previous antitumor therapy - \# (\%) } \\
\hline Radiation \pm Resection & $26(96.3)$ & $11(100)$ & \\
\hline Chemotherapy \pm Resection & $13(48.1)$ & $5(45.5))$ & NS \\
\hline \multicolumn{4}{|l|}{ Number of previous surgeries - \# (\%) } \\
\hline 1 & $20(74.1)$ & $9(81.8)$ & \\
\hline 2 & $5(18.5)$ & $2(18.2)$ & NS \\
\hline$>2$ & $2(7.4)$ & $0(0.0)$ & \\
\hline \multicolumn{4}{|c|}{ Number of previous radiotherapies - \# (\%) } \\
\hline 0 & $1(3.7)$ & $0(0.0)$ & \\
\hline 1 & $24(88.9)$ & $10(90.9)$ & NS \\
\hline 2 & $2(7.4)$ & $1(9.1)$ & \\
\hline \multicolumn{4}{|l|}{ Number of previous chemotherapies } \\
\hline 0 & $14(51.9)$ & $6(54.5)$ & \\
\hline $\begin{array}{l}1 \\
2\end{array}$ & $\begin{array}{c}13(48.1) \\
0(0.0)\end{array}$ & $\begin{array}{c}4(36.4) \\
1(9.1)\end{array}$ & NS \\
\hline $\begin{array}{l}\text { Best Overall Response } \\
\text { CR }+ \text { PR } \\
\text { No objective response }\end{array}$ & $\begin{array}{l}14 \\
13\end{array}$ & $\begin{array}{l}1 \\
9\end{array}$ & $\begin{array}{c}\mathbf{P}=\mathbf{0 . 0 2 8} \\
\text { (Fisher's Exact, } \\
\text { two-tailed) }\end{array}$ \\
\hline
\end{tabular}

NA: not applicable; NS: P-value $>0.05 /$ not significant. ${ }^{*}$ One of the 11 TMZ-treated patients died early due to pneumonia on day 16 after randomization and was not evaluable for best overall response 


\section{Statistical analyses}

Standard statistical methods were applied for the analysis of data. The distribution of time-to-event survival end points on the OS and PFS curves were estimated by the Kaplan-Meier method. Differences between patient subgroups were evaluated by log-rank statistics. The analyses were performed using JMP software (version 10.02, SAS Institute, Inc, Cary, NC), and R software, version 3.5.2 (R Foundation for Statistical Computing) loaded with statistical packages for survival analysis (survMisc_0.5.5; survival_2.44-1.1 and survminer_0.4.4) with default settings. Survival curves were visualized using the survminer graphing package (Drawing Survival Curves using 'ggplot2'. R package version 0.4.4; https://CRAN.R-project.org/package=survminer). For the patients who had a CR or PR as their BOR, Waterfall plots were used to represent the maximum percentage or log10 change in MRIbased tumor volume of the target lesion relative to measurements taken at baseline. To test whether a fixed fraction of the tumor cells is killed regardless of the tumor size, we investigated the first order kinetics of the tumor reductions in each of the objective responder patients (viz., patients with a CR or PR as their BOR) by fitting a straight line to a semi-log plot of the portion of the tumor growth curve that displayed maximum reduction in tumor size over the course of OT101 treatment. The slope of the line represents the rate constant for tumor reduction in $\log 10$ scale, and times to $90 \%$ (T10: $-1 /$ Slope) reduction of tumor volumes were calculated using the rate constant.

\section{Results and discussion}

Fourteen of the 27 patients (51.9\%) had a sustained partial response (PR) or complete response (CR) (Table 2) to the immuno-oncology drug OT101 following a robust size reduction of their target lesions (Figure 2). The median time for $90 \%$ reduction of their baseline tumor volume was 11.8 months (Range: 4.9-57.7 months). Seven (50\%) of the 14 responders were in the $2.5 \mathrm{mg} / \mathrm{cycle}$ low dose cohort and 7 (50\%) were in the $19.8 \mathrm{mg} /$ cycle high dose cohort. Waterfall plots depicting the maximum $\log 10$ reduction values for the tumor volumes of the 14 objective responders following OT101 treatment are shown in Figure 3. The mean $\log 10$ reduction for the 14 responders was $2.2 \pm 0.4$ (Median=1.6, Range: 0.4-4.3) logs. The mean percent reduction for the 14 responders was $92.6 \pm 3.4$ (Median=97.0, Range: $57.5-100 \%$ ). Four patients achieved $100 \%$ reduction in tumor volume over the course of the treatment.

The median time to objective response was 274 (Range: 37-914) days (mean \pm SE : $322 \pm 56$ days). The PR in 2 of these 14 patients deepened to a CR at 917 and 1838 days, respectively. Of these 14 objective responders, 10 had a response duration of $>6$ months, including 3 patients with a response duration of 7.3-9.4 months and 5 patients had a durable response with a median response duration of 3.7 years (viz.: 1.1 years, 3.0 years, 3.7 years, 3.8 years, and 4.2 years. By comparison, only one of the 10 control patients with Grade 3 AA evaluable for response who were treated with the alkylating chemotherapy drug temozolomide (TMZ) had an objective response/PR (10\%) $(\mathrm{P}=0.028$, Fisher's Exact test, two-tailed) and the PR lasted only 142 days (4.7 months).

This highly significant difference in objective response rates also translated into an overall survival advantage for the OT101-treated AA patients regardless of the dose cohort. For the 27 patients treated with OT101, the Kaplan-Meier probability estimates for the median OS was 1136 (95\% CI: 811 - 1743) days which was significantly better than the 590 (95\% CI: 287 - NA) days median OS (Log Rank $\chi^{2}=6.5$, $\mathrm{P}$-value $=0.011$ ) of the TMZ-treated control patient population (Figure 4). There were no differences in survival outcome of the two OT101 dose cohorts: Median OS=1262 days (95\% CI: 963-NA days) for 2.5 $\mathrm{mg} /$ cycle $(\mathrm{N}=12)$ vs Median OS=1072 days (95\% CI: $424-\mathrm{NA}$ days) for $19.8 \mathrm{mg} /$ cycle, Log Rank $\left.\chi^{2}=0.49, \mathrm{P}=0.49\right)$. These results in $\mathrm{R} / \mathrm{R}$ AA patients demonstrate that CEDOT-administered OT101 induces durable $\mathrm{PR}$ or $\mathrm{CR}$ in an aggressive brain tumor patient population. To our knowledge, this is the first demonstration that the intratumoral delivery of an RNA therapeutic (or any other immuno-oncology drug candidate) via extended CED in the absence of other therapeutic agents or radiation results in $>3.5$ year OS in more than half of a R/R highgrade glioma patient population. The CEDOT platform is anticipated to allow the combined use of distinct immuno-oncology modalities, including immune checkpoint inhibitors, oncolytic viruses, and antiTGF agents with a high clinical impact potential for aggressive and difficult-to-treat brain tumors.

A number of limitations should be pointed out for this study: First and foremost, our results should be interpreted with due caution within the inherent limitations of a post-hoc analysis. Having an objective response (i.e., CR or PR) is not a proven surrogate marker for PFS or OS in HGG patients [17]. Notwithstanding the fact that MRI imaging provides only a surrogate measure of the disease activity and status in HGG patients [17], the observed association of the objective responses with prolonged OS demonstrates that OT101 treatments can benefit R/R HGG patients. The observed robust tumor reduction in a subgroup of patients with favourable responses as confirmed by independent central review combined with the long-term survival of objective responders is unprecedented for an immuno-oncology drug. Other limitations of this Phase II study include the heterogeneous nature, lack of genomic information and very small size for the patient population. It will be important to further evaluate the clinical potential of OT101 in a larger and a more homogenous patient population (e.g. IDHwildtype WHO Grade 3 AA patients who have failed a temozolomidebased first line adjuvant therapy, especially those whose tumors are MGMT-unmethylated).

In the present study, OT101 was administered via CED [11] and a number of CED and device-related complications were identified. It will be important to reduce the risk of procedure/device related early complications, including CNS infections to not only maximize patient safety but also to further improve the OS outcome. The use of antibioticimpregnated catheters that have recently become commercially available and implementation of an intensive training program for the medical personnel involved in the maintenance of the CED system to ensure high standards of hygiene as well as perioperative use of prophylactic antibiotics during catheter and port placement may reduce the risk of infections. Additional risk mitigation measures may help avoid potential catheter misplacement and intratumoral bleeding in future studies by (i) avoiding biopsies from the catheter tip which might lead to tissue disruption as well as bleeding and (iii) applying high precision catheter placement by an optimized stereotactic procedure and by tracking the delivery of the OT101 infusion using real-time intraoperative MRI imaging as well as using a software for 3-D drug distribution simulation. We anticipate that the incorporation of the insights and lessons learned from this analysis in the further clinical evaluation and development of OT101 will help improve the potential for patient benefit.

TGF $\beta$ has been shown curb the anti-tumor function of TME by both limiting T-cell infiltration and suppressing the function of the immune system elements [18-21]. TGF $\beta$ has been implicated as a key contributor to the immunosuppressive landscape of the TME in HGGs $[22,23]$. TGF $\beta$ has recently been implicated in T-cell exclusion from TME contributing to tumor immune evasion and a poor response to 


\section{UPN403405}
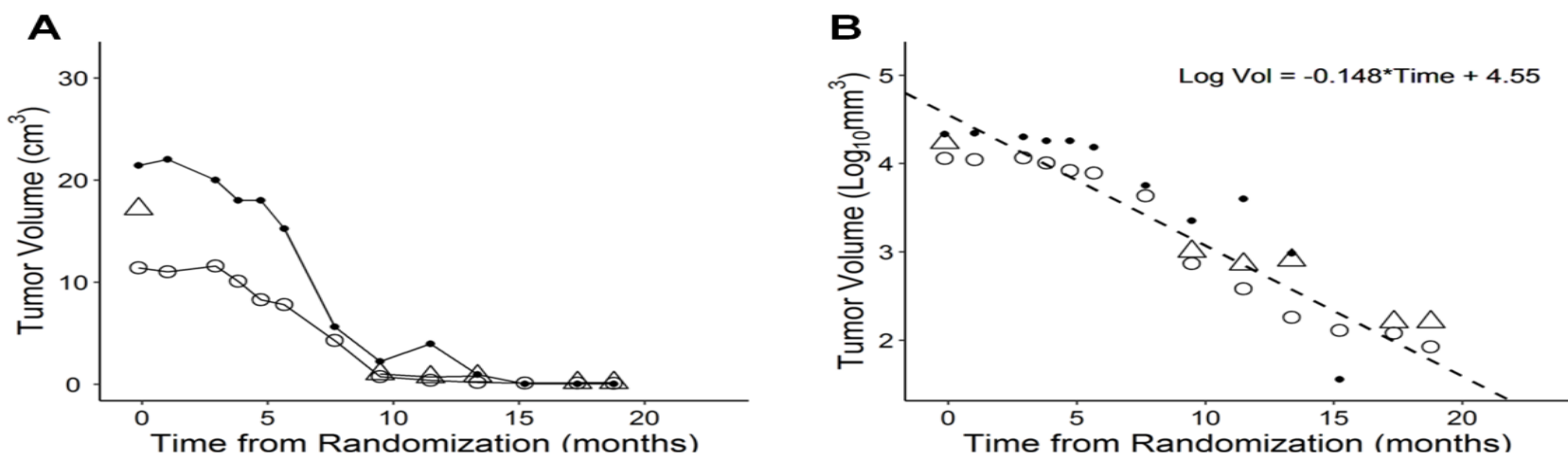

UPN407409
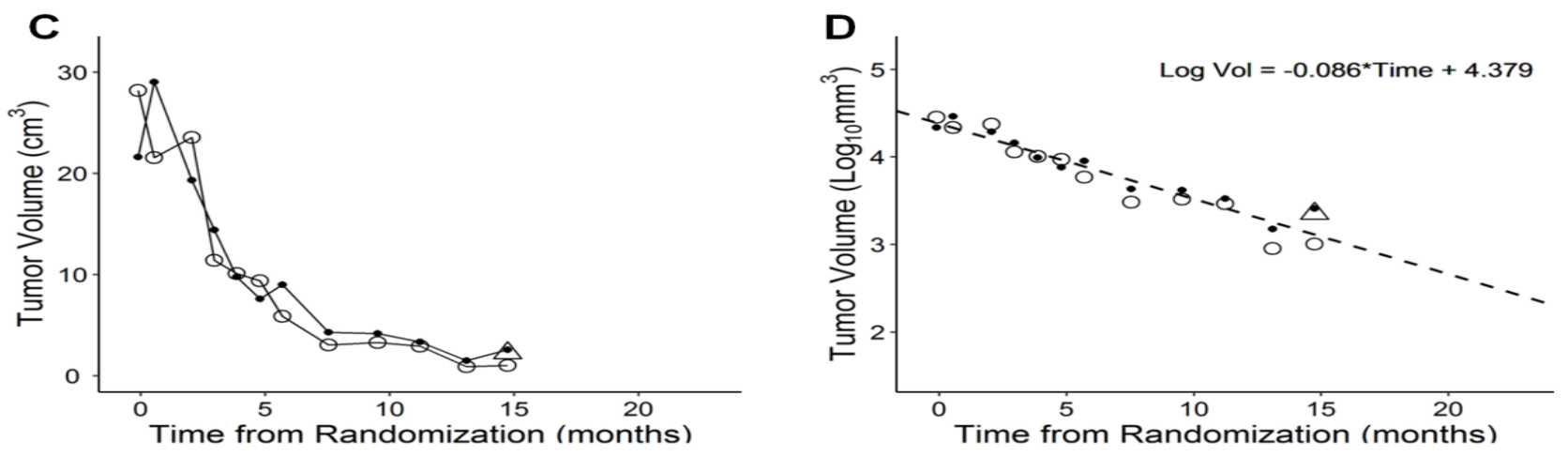

\section{UPN405413}
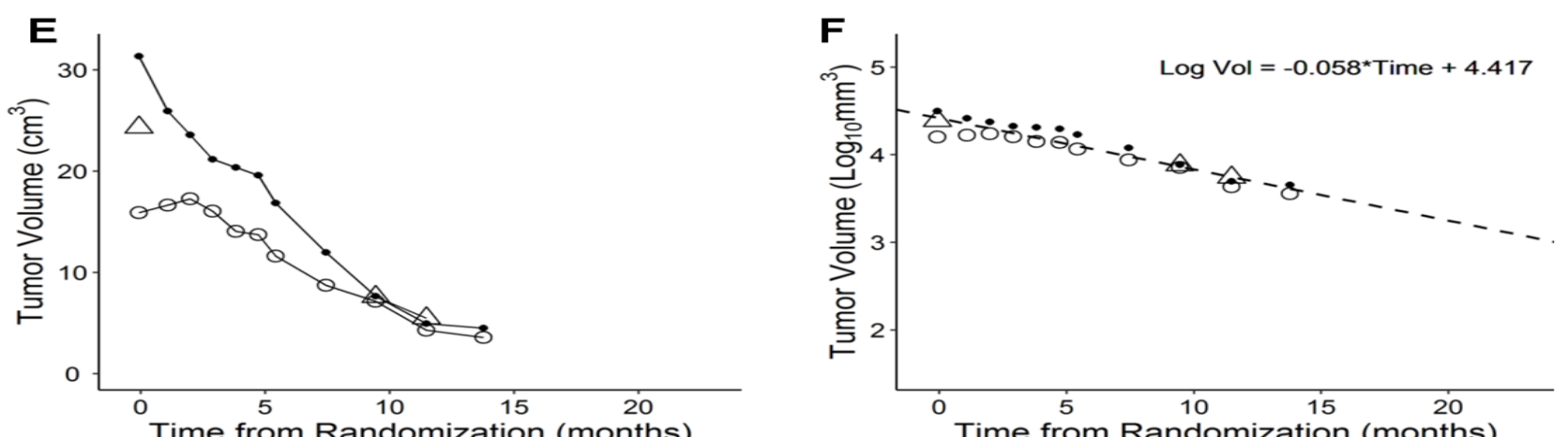

Figure 2. Time-dependent reduction of target lesion size in OT101 treated R/R adult AA (WHO Grade III) patients. These 3 representative AA patients achieved an objective response by standard McDonald criteria. In all 3 patients, review of MRI images by 2-3 independent reviewers (open circle: Reviewer 1; Closed circle: Reviewer 2; Triangle: Reviewer 3/ Adjudicator) showed a time-dependent decrease of the 3-D (Panels A, C, E) size of the target lesion. We also investigated the first order kinetics of the tumor reductions in each patient by fitting a straight line to a semi-log plot of the portion of the 3-D tumor volume reduction curve that displayed maximum reduction in tumor size over the course of OT101 treatment (Panels B, D and F). The slope of the line represents the rate constant for tumor reduction in $\log 10$ scale, and duration times to $90 \%$ (T10: $-1 / \mathrm{Slope}$ ) and $99 \%$ (T1: -2/Slope) percent reduction of tumor volumes were calculated using the rate constant 

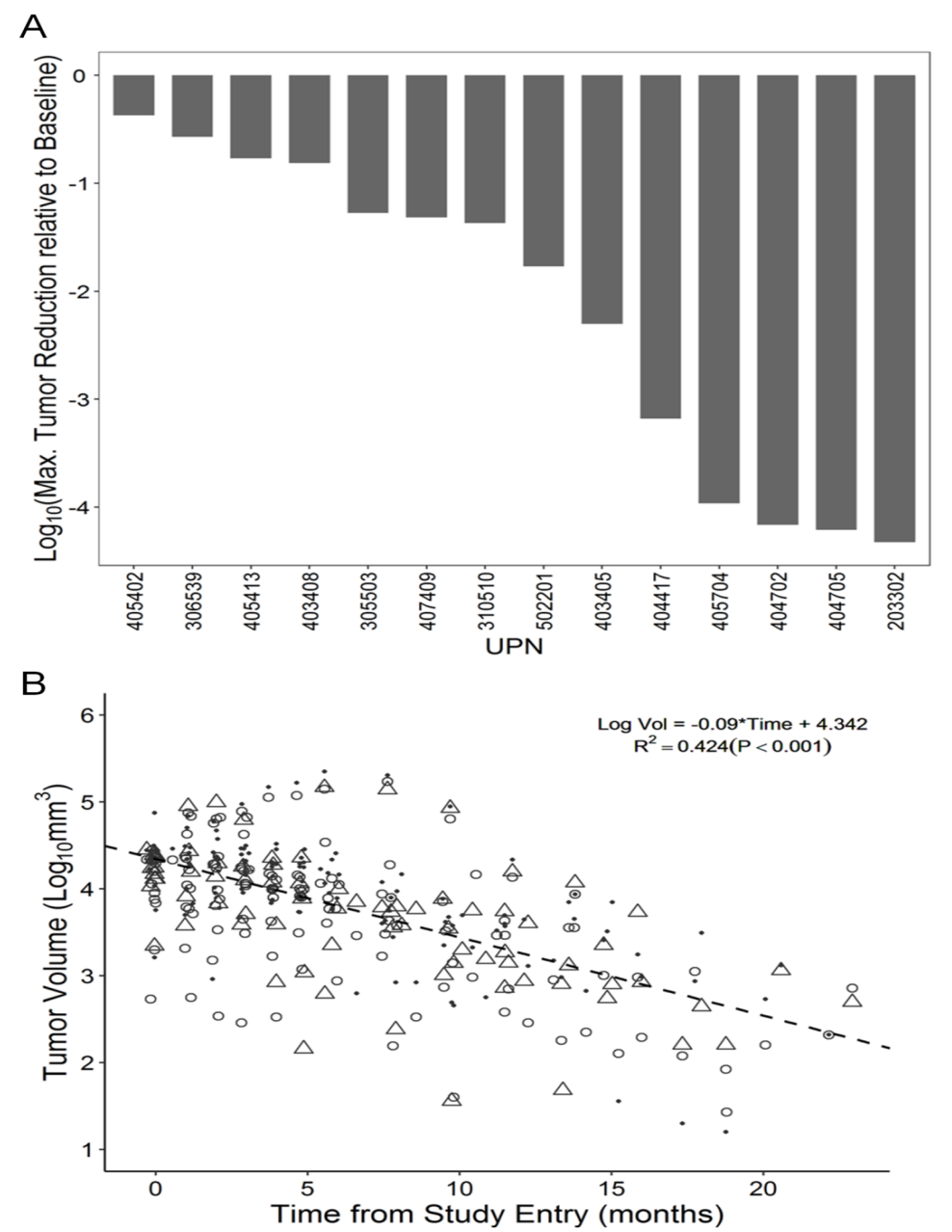

Figure 3. Imaging Responses in R/R AA (WHO Grade 3) Patients Treated with OT101 Monotherapy Who Achieved a CR or PR. [A] A waterfall plot depicting the maximum log 10 reduction values for the tumor volumes. [B] A semi-log plot of the combined 3-D tumor volume reduction curve for the 14 patients with a CR or PR. The first order kinetics of the tumor volume reduction for the entire population of the 14 objective responders is illustrated by fitting a straight line to a semi-log plot of the portion of the tumor reduction curve that displayed maximum reduction in tumor size over the course of OT101 treatment. Data points represent the individual assessments from 2-3 radiologists for each time point of MRI assessment for each of the 14 patients

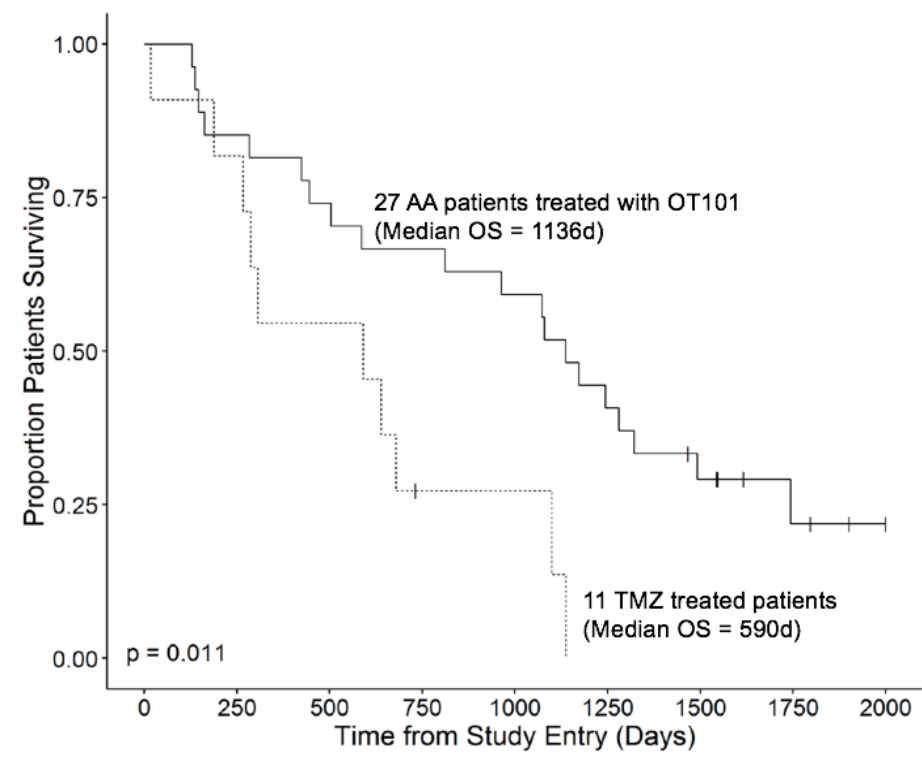

Figure 4. Overall survival Outcome of R/R AA patients according to their treatment group. Depicted are the OS curves of the entire 27-patient OT101-treated population and 11-patient TMZ-treated population 
immune checkpoint inhibitors [18-21]. TGF $\beta$ is being explored as a therapeutic target for the treatment of HGGs, due to the compelling evidence that the amplified activity of the TGF $\beta$-SMAD signalling pathway contributes to the malignant phenotype and poor prognosis of GBM in adult patients by enhancing tumor growth, invasion, and angiogenesis as well as compromised immune surveillance $[12,13,24-$ 30]. These recent observations regarding the role of TGF $\beta$ in tumorinduced immune suppression prompt the hypothesis that OT101 could provide the basis for a combined treatment strategy of targeting both TGF $\beta$ and PD-1/PD-L1 pathways in recurrent HGG patients. Intratumoral infusion of a recombinant polio-rhinovirus chimera has also shown a promising clinical activity signal in a recent clinical study [31]. The CEDOT platform would seem suitable to deliver rationally designed combinations of promising agents $[1,7,31,32]$ as salvage therapy for R/R HGG patients.

\section{Author contributions}

All authors have contributed to the analysis of data. The corresponding author designed the evaluations reported in this paper, directed the data compilation and analysis, and prepared the initial draft of the manuscript. Each author had access to the source data used in this post-hoc analysis. Each author reviewed and revised the manuscript, provided final approval for submission of the final version. No medical writer was involved.

\section{References}

1. Jain KK (2018) A critical overview of targeted therapies for glioblastoma. Frontiers in Oncology 8: 1-19.

2. Stupp R, Hegi ME, Mason WP (2009) Effects of radiotherapy with concomitant and adjuvant temozolomide versus radiotherapy alone on survival in glioblastoma in a randomised phase III study: 5-year analysis of the EORTC-NCIC trial. Lancet Oncol 10: $459-466$.

3. Stupp R, Taillibert S, Kanner AA, Kesari S, Steinberg DM, et al. (2015) maintenance therapy with tumor-treating fields plus temozolomide vs temozolomide alone for glioblastoma: A randomized clinical trial. JAMA 314: 2535-2543. [Crossref]

4. Herrlinger U, Tzaridis T, Mack F (2019) Lomustine-temozolomide combination therapy versus standard temozolomide therapy in patients with newly diagnosed glioblastoma with methylated MGMT promoter (CeTeG/NOA-09): a randomised, open-label, phase 3 trial. Lancet 393: 678. [Crossref]

5. Lieberman F (2017) Glioblastoma update: molecular biology, diagnosis, treatment, response assessment, and translational clinical trials. F1000Res 6: 1892. [Crossref]

6. Miller JJ, Wen PY (2016) Emerging targeted therapies for glioma. Expert Opin Emerg Drugs 21: 441-452.

7. Carlsson SK, Brothers SP, Wahlestedt C (2014) Emerging treatment strategies for glioblastoma multiforme. EMBO Mol Med 6: 1359-1370.

8. van Bussel MTJ, Beijnen JH, Brandsma D (2019) Intracranial antitumor responses of nivolumab and ipilimumab: a pharmacodynamic and pharmacokinetic perspective, a scoping systematic review. BMC Cancer 19: 519.

9. Kamath SD, Kumthekar PU (2018) Immune Checkpoint inhibitors for the treatment of central nervous system (CNS) metastatic disease. Front Oncol 8: 414.

10. Lauko A, Thapa B, Jia X (2018) Ahluwalia MS: Efficacy of immune checkpoin inhibitors in patients with brain metastasis from NSCLC, RCC, and melanoma. J Clin Oncol 36: 214-214.

11. Jahangiri AJ, Chin AT, Flanigan PM, Chen R, Bankiewicz K (2017) Convectionenhanced delivery in glioblastoma: a review of preclinical and clinical studies. $J$ Neurosurg 126: 191-200
12. Hau P, Jachimczak P, Bogdahn U (2009) Treatment of malignant gliomas with TGFbeta2 antisense oligonucleotides. Expert Rev Anticancer Ther 9: 1663-1674.

13. Luc Vallières (2009) Trabedersen, a TGFß2-specific antisense oligonucleotide for the treatment of malignant gliomas and other tumors overexpressing TGFß2. IDrugs 12 445-453.

14. Hau P, Jachimczak P, Schlingensiepen R (2007) Inhibition of TGF-beta2 with AP 12009 in recurrent malignant gliomas: from preclinical to phase I/II studies. Oligonucleotides 17: 201-212.

15. Schlingensiepen R, Goldbrunner M, Szyrach MNI (2005) Intracerebral and intrathecal infusion of the TGF-beta2-specific antisense phosphorothioate oligonucleotide AP 12009 in rabbits and primates: toxicology and safety. Oligonucleotides 15: 94-104.

16. Bogdahn U, Hau P, Stockhammer (2011) Targeted therapy for high-grade glioma with the TGF-beta2 inhibitor trabedersen: Results of a randomized and controlled phase IIb study. Neuro-Oncology 13: 132-142.

17. Ellingson BM, Wen PY, Cloughesy TF (2017) Evidence and context of use for contrast enhancement as a surrogate of disease burden and treatment response in malignant glioma. Neuro-Oncology 20: 457-471

18. Tauriello DVF, Palomo-Ponce S, Stork D (2018) TGFÎ drives immune evasion in genetically reconstituted colon cancer metastasis. Nature 554: 538-543. [Crossref]

19. Ganesh K, Massagué J (2018) TGF- $\beta$ Inhibition and Immunotherapy: Checkmate. Immunity 48: 626-628.

20. Mariathasan S, Turley SJ, Nickles D, Castiglioni A, Yuen K, et al. (2018) TGFî attenuates tumour response to PD-L1 blockade by contributing to exclusion of T cells Nature 554: 544-548. [Crossref]

21. Thomas DA, Massagué J (2005) TGF- $\beta$ directly targets cytotoxic T cell functions during tumor evasion of immune surveillance. Cancer Cell 8: 369-380.

22. Brooks WH, Netsky MG, Normansell DE, Horwitz DA (1972) Depressed cell-mediated immunity in patients with primary intracranial tumors. Characterization of a humoral immunosuppressive factor. $J$ Exp Med 136: 1631-1647.

23. Kuppner MC, Hamou MF, Sawamura Y, Bodmer S, De Tribolet N (1989) Inhibition of lymphocyte function by glioblastoma-derived transforming growth factor beta $2 . J$ Neurosurg 71: 211-217.

24. Han J, Alvarez-Breckenridge CA, Wang QE, Yu J (2015) TGF-ß signaling and its targeting for glioma treatment. Am J Cancer Res 5: 945-55.

25. Kjellman C, Olofsson SP, Hansson O, Von Schantz T, Lindvall M, et al. (2000) Expression of TGF-beta isoforms, TGF-beta receptors, and SMAD molecules at different stages of human glioma. Int J Cancer 89: 251-258. [Crossref]

26. Fakhrai H, Dorigo O, Shawler DL, Lin H, Mercola D, et al. (1996) Eradication of established intracranial rat gliomas by transforming growth factor beta antisense gene therapy. Proc Natl Acad Sci U S A 93: 2909-2914. [Crossref]

27. Frei K, Gramatzki D, Tritschler I, Schroeder JJ, Espinoza L (2015) Transforming growth factor- $\beta$ pathway activity in glioblastoma. Oncotarget 6: 5963-5977.

28. Roy LO, Poirier MB, Fortin D (2015) Transforming growth factor-beta and its implication in the malignancy of gliomas. Target Oncol 10: 1-14.

29. Peñuelas S, Anido J, Prieto-Sanchez RM (2009) TGF-beta Increases Glioma-Initiating Cell Self-Renewal through the Induction of LIF in Human Glioblastoma. Cancer Cell 15: 315-327.

30. Bruna A, Darken RS, Rojo F (2007) High TGFß-Smad activity confers poor prognosis in glioma patients and promotes cell proliferation depending on the methylation of the PDGF-B gene. Cancer Cell 11: 147-160.

31. Desjardins A, Gromeier M, Herndon JE (2018) Recurrent Glioblastoma Treated with Recombinant Poliovirus. N Engl J Med 379: 150-161.

32. Chandramohan V, Bao X, Yu X (2019) Improved efficacy against malignant brain tumors with EGFRwt/EGFRvIII targeting immunotoxin and checkpoint inhibitor combinations. J Immunother Cancer 7: 142.

Copyright: (C2020 Uckun FM. This is an open-access article distributed under the terms of the Creative Commons Attribution License, which permits unrestricted use, distribution, and reproduction in any medium, provided the original author and source are credited. 